Predi cti on of di scont i nuous change of vol une, ent ropy, and heat capacity at mel ting transition i n crystal I i ne pol ymers based on the Landau theory

\begin{tabular}{|l|l|}
\hline 著者 & SAEK S \\
\hline $\begin{array}{l}\text { j our nal or } \\
\text { publ i cat i on t i t l e }\end{array}$ & FI ui d Phase Equi I i bri a \\
\hline vol une & 235 \\
\hline nunber & 2 \\
\hline page r ange & $166-172$ \\
\hline year & $2005-08$ \\
\hline URL & ht t p: //hdl . handl e. net /10098/1162 \\
\hline
\end{tabular}




\title{
Prediction of discontinuous change of volume, entropy, and heat capacity at melting transition in crystalline polymers based on the Landau theory
}

\author{
S. Saeki* \\ Department of Materials Science and Engineering, Fukui University, Fukui 910 8507, Japan
}

\begin{abstract}
The discontinuous changes of volume $\Delta V_{\mathrm{m}}$, entropy $\Delta S_{\mathrm{m}}$ and heat capacity at constant pressure $\Delta C_{P, \mathrm{~m}}$ at the melting point have been predicted by the Landau theory of phase transition where the Gibbs free energy $\Phi$ in order state is expressed by $\Phi=\Phi_{0}+\mathrm{A} \eta^{2}-\mathrm{C} \eta^{4}+\mathrm{E} \eta^{6}$, where $\eta$ is an order parameter. In this work, $A$ is given by a function that $A=k_{0}\left(T-T_{0}\right)^{m}\left(P_{0}-P\right)^{n}$, where $k_{0}, m, n, T_{0}$, and $P_{0}$ are constants. The order parameter $\eta_{\min }$ for the minimum of $\Phi_{\min }$ is related to the volume of solid $V_{s}$ and is expressed by:

$\left(\frac{C}{A^{*}}\right)^{2} \eta_{\min }^{4}-\left(\frac{3}{8}\right)\left(\frac{C}{A^{*}}\right)^{3} \eta_{\min }^{6}=1-\frac{\Delta V}{\Delta V_{\mathrm{m}}}$

where $\Delta V=V_{\mathrm{s}}-V_{\mathrm{m}, \mathrm{s}}, \Delta V_{\mathrm{m}}=V_{\mathrm{m}, 1}-V_{\mathrm{m}, \mathrm{s}}$ and $A^{*}$ is the value of $A$ at the melting point. The volume of $V_{\mathrm{s}}$ changes from $V_{\mathrm{m}, \mathrm{s}}$ to $V_{\mathrm{m}, 1}$, where $\Delta V_{\mathrm{m}, s}$ and $\Delta V_{\mathrm{m}, 1}$ are volumes of solid and liquid phases at the melting point, respectively. The discontinuous change of thermal pressure coefficient $\gamma_{V}=(\partial P / \partial T)_{V}$, thermal expansion $(\partial V / \partial T)_{P}$ and compressibility $-(\partial V / \partial P)_{T}$ at the melting point has been calculated. A typical function of $\Phi$ for a polymer has been demonstrated as a function of $\eta$ and $C$ at the melting transition.
\end{abstract}

(c) 2005 Elsevier B.V. All rights reserved.

Keywords: Melting transition; Landau theory; Crystalline polymer; Order parameter

\section{Introduction}

The melting phenomena of crystalline polymer are a process involving the disorderly arrangement of polymer chains and are consequently associated with evolution of large positive entropy $\triangle S_{\mathrm{m}}$ which is accompanied with the volume change $\Delta V_{\mathrm{m}}$ under constant temperature and pressure. Investigation of discontinuous change of thermodynamic quantities at the melting temperature $T_{\mathrm{m}}$ of crystalline polymers is of great importance in polymer processing. When a manufacturing product of crystalline polymer is made from polymer melt, the polymer passes through the melting and crystallization in the process. Among important thermody-

* Tel.: +81776278622 ; fax: +81776278767 .

E-mail address: sacki@matse.fukui-u.ac.jp. namic quantities of polymer, the thermal pressure coefficient $\gamma_{V}=(\partial P / \partial T)_{V}$ is a key factor to control of size of product in the crystallization process. In addition to the $\gamma_{v}$, the discontinuous changes of heat capacity $C_{P}$ at constant pressure, thermal expansion $(\partial V / \partial T)_{P}$ and compressibility $-(\partial V / \partial P)_{T}$ are also important to control the polymer processing. In this work, we have investigated the behavior of discontinuous changes of thermodynamic quantities at the melting transition by the Landau theory $[1,2]$.

\section{Prediction of discontinuous change at the melting point based on the Landau theory}

A concept of the Landau theory of phase transition rests on an assumption that the Gibbs free energy function over 
a range of phase transition is expressed by introducing an order parameter in addition to the fundamental thermodynamic variables of pressure $P$ and temperature $T$. According to the Landau theory $[1,2]$, the Gibbs free energy function $\Phi$ in order state is expressed by:

$\Phi=\Phi_{0}+A \eta^{2}-C \eta^{4}+E \eta^{6}$

where $\eta$ is the order parameter and $A$ is a function of temperature and pressure, while $C$ and $E$ are assumed to be independent of pressure and temperature in this work. One of the main reasons for the assumption is that if $C$ and $E$ are dependent of pressure and temperature and extra coefficients for $C$ and $E$ are introduced, we cannot determine the $\Phi$ function by the five experimental data of discontinuous changes. In this equation conditions that $C$ and $E$ are positive and $A$ is positive are necessary conditions to have two minimum points in $\Phi$ versus $\eta^{2}$ curve where the minimum points correspond to the coexisting two phases. It is found experimentally that values of $C$ and $E=C^{2} / 4 A^{*}$ are positive as is shown later. The value of $\Phi_{0}$ is the Gibbs free energy function $\Phi$ for non-ordered state with $\eta=0$. The thermodynamic stable state is given by the minimum condition of free energy with respect to $\eta$ and $(\partial \Phi / \partial \eta)=0$. In Eq. (1), the condition leads to two equations that $\eta=0$ and the other is a meaningful one that:

$A-2 C \eta^{2}+3 E \eta^{4}=0$

The expression of $\Phi_{\min }$ is given using Eqs. (1) and (2) by:

$\Phi_{\min }=\Phi_{0}+\left(\frac{4 A^{* 2}}{27 C}\right)\left(4-3 Z^{2}-Z^{3}\right)$

The parameter $Z$ in Eq. (3) is expressed by:

$Z=\left(4-\frac{3 A}{A^{*}}\right)^{1 / 2}$

where $A^{*}$ is the value of $A$ at the melting point $\left(P_{\mathrm{m}}, T_{\mathrm{m}}\right)$. The derivation of Eq. (3) is given in Appendix $A$ in more detail. The parameter $Z$ is related to the order parameter $\eta_{\min }$ by:

$\eta_{\min }^{2}=\left(\frac{2 A^{*}}{3 C}\right)(2+Z)$

The melting point $\left(P_{\mathrm{m}}, T_{\mathrm{m}}\right)$ is a point determined by both conditions that $(\partial \Phi / \partial \eta)=0$ and $\Phi_{\min }\left(\eta_{\min }, P_{\mathrm{m}}, T_{\mathrm{m}}\right)=\Phi_{0}$, where $\Phi_{\min }\left(\eta_{\min }, P_{\mathrm{m}}, T_{\mathrm{m}}\right)$ corresponds to $\Phi_{\min }$ for the crystalline or solid phase coexisted with the liquid one with $\Phi_{0}$. The function of $A$ with respect to $P$ and $T$ is essentially important in the Landau theory. In this work, we have assumed the function $A$ by:

$$
A=k_{0}\left(T-T_{0}\right)^{m}\left(P_{0}-P\right)^{n}
$$

where $k_{0}, m$, and $n$ are constant and $T_{0}$ and $P_{0}$ are characteristic temperature and pressure at $A=0$. The $A^{*}$ is the value of $A$ at the melting point and is given by
$A^{*}=k_{0}\left(T_{\mathrm{m}}-T_{0}\right)^{m}\left(P_{0}-P_{\mathrm{m}}\right)^{n}$. A brief summary of possible forms of the $A(P, T)$ function could be appropriate in order to understand our choice. A possibility of other type of function $A$, such as $A=a\left(T-T_{0}\right)+b\left(P_{0}-P\right)$ is discussed later.

The volume and entropy at the melting point is derived from the Eq. (3) by using the thermodynamic relation that $\left(\partial \Phi_{\min } / \partial P\right)_{T}=V$ and $\left(\partial \check{\Phi}_{\min } / \partial T\right)_{P}=-S$, respectively. For examples, the volume in order state or solid state $V_{\mathrm{S}}$ is given using Eq. (3) by:

$$
V_{\mathrm{s}}=V_{\mathrm{m}, 1}-\frac{\left(2 A^{* 2} n / 9 C\right)(2+Z)\left(4-Z^{2}\right)}{P-P_{0}}
$$

The volume of solid coexisted with liquid at $T_{\mathrm{m}}$ is defined by $V_{\mathrm{m}, \mathrm{s}}$ and is given by $V_{\mathrm{s}}$ in Eq. (7) with $Z=1$. The discontinuous volume change $\Delta V_{\mathrm{m}}$ at the melting point $T=T_{\mathrm{m}}$ and $P=P_{\mathrm{m}}$ is given using Eq. (7) by:

$\Delta V_{\mathrm{m}}=V_{\mathrm{m}, 1}-V_{\mathrm{m}, \mathrm{s}}=\left(\frac{2 A^{* 2} n}{C\left(P_{0}-P_{\mathrm{m}}\right)}\right)$

where $V_{\mathrm{m}, 1}$ is the volume for liquid phases at the melting point.

The melting phenomena occur when temperature of system approaches to the melting temperature at constant pressure. The discontinuous volume change at the melting is due to the transformation from the solid to liquid where the order parameter $\eta$ changes with volume drastically. The order parameter $\eta_{\min }$ for the minimum of $\Phi_{\min }$ is expressed as a function volume of solid $V_{\mathrm{S}}$, which is given using Eqs. (5), (7) and (8) at $P=P_{\mathrm{m}}$ by:

$$
\left(\frac{C}{A^{*}}\right)^{2} \eta_{\min }^{4}-\left(\frac{3}{8}\right)\left(\frac{C}{A^{*}}\right)^{3} \eta_{\min }^{6}=1-\frac{\Delta V}{\Delta V_{\mathrm{m}}}
$$

where $\Delta V$ is defined by $\Delta V=V_{\mathrm{s}}-V_{\mathrm{m}, \mathrm{s}}$ and is obtained by $\Delta V=V_{\mathrm{s}}-V_{\mathrm{m}, \mathrm{l}}+V_{\mathrm{m}, 1}-V_{\mathrm{m}, \mathrm{s}}$. The function of $\left(V_{\mathrm{s}}-V_{\mathrm{m}, \mathrm{l}}\right)$ is calculated from Eq. (7) and $V_{\mathrm{m}, 1}-V_{\mathrm{m}, \mathrm{s}}$ is by Eq. (8). The $\Delta V$ is not difference in the volumes of two phases. The variable $\Delta V\left(=V_{\mathrm{s}}-V_{\mathrm{m}, \mathrm{s}}\right)$ is related to $V_{\mathrm{s}}$ directly and changes from $\Delta V=0$ for $V_{\mathrm{s}}=V_{\mathrm{m}, \mathrm{s}}$ at the beginning of melting transition to $\Delta V=\Delta V_{\mathrm{m}}\left(=V_{\mathrm{m}, 1}-V_{\mathrm{m}, \mathrm{s}}\right)$ for $V_{\mathrm{s}}=V_{\mathrm{m}, 1}$ at the end of melting.

The experimental values of discontinuous changes, such as $\Delta S_{\mathrm{m}}, \Delta V_{\mathrm{m}}, \Delta C_{P, \mathrm{~m}},-\Delta(\partial V / \partial P)_{T, \mathrm{~m}}$ and $\Delta(\partial V / \partial T)_{P, \mathrm{~m}}$ at the melting point are listed in Table 1 for nine crystalline polymers where all these values are positive for the nine polymers. This indicates that values of $Q_{\mathrm{m}, 1}$ at the liquid phase are larger than $Q_{\mathrm{m}, \mathrm{s}}$ at solid phase at the melting point, where $Q$ corresponds to the quantity such as $S$ and $V$. The relations between the discontinuous changes $\Delta Q_{\mathrm{m}}$ and the coefficients or indices such as $C, A^{*}, m$, and $n$ in the Eqs. (1) and (6) are summarized in Table 2 . The qualitative relations between the indices of $m$ and $n$ in the function of $A$ and the sign of 
Table 1

The experimental data of gap at the phase transition and indices of $m$ and $n$ calculated by Eqs. (10) and (11) for some crystalline polymers

\begin{tabular}{|c|c|c|c|c|c|c|c|c|c|c|}
\hline & $\begin{array}{l}T_{\mathrm{m}}(\mathrm{K}, \\
\left.P_{\mathrm{m}}=1 \text { bar }\right)\end{array}$ & $\begin{array}{l}\Delta S_{\mathrm{m}} \\
(J / \mathrm{K} \mathrm{mol})\end{array}$ & $\begin{array}{l}\Delta V_{\mathrm{m}} \\
(\mathrm{J} / \mathrm{bar} \mathrm{mol})\end{array}$ & $\begin{array}{l}\Delta C_{P, \mathrm{~m}} \\
(\mathrm{~J} / \mathrm{K} \mathrm{mol})\end{array}$ & $\begin{array}{l}-\Delta\left(\frac{\partial V}{\partial P}\right)_{T, \mathrm{~m}} \\
\left(10^{-5} \mathrm{~J} / \mathrm{bar}^{2}\right)\end{array}$ & $\begin{array}{l}\Delta\left(\frac{\partial V}{\partial T}\right)_{P, m} \\
\left(10^{-4} \mathrm{~J} / \mathrm{barK}\right)\end{array}$ & $\begin{array}{l}\frac{A^{* 2}}{C} \\
\left(10^{3} \mathrm{~J} / \mathrm{mol}\right)\end{array}$ & $m$ & $n$ & Ref. \\
\hline $\mathrm{PE}$ & $411,414.6$ & $9.58,9.88$ & $0.287,0.355$ & $6.28,9.0$ & $11.9,4.2$ & $7.0,9.3$ & $0.98,0.94$ & $5.8,12.4$ & $-0.4,-7.8$ & {$[3,4,11]$} \\
\hline $\mathrm{PP}$ & $449,452.4$ & $17.6,15.1$ & $0.714,0.594$ & $17.57,12.0$ & $13.8,6.3$ & $21.8,25.2$ & $1.44,0.90$ & $7.3,3.4$ & $-3.5,5.5$ & {$[3,4,10]$} \\
\hline PB-1 & 411.2 & 15.9 & 0.75 & 3.0 & 2.1 & 13.7 & 2.18 & 2.67 & 2.96 & {$[3,10]$} \\
\hline POM & 457.2 & 21.0 & 0.436 & 10.0 & 4.4 & 12.9 & 1.77 & 3.1 & -3.1 & {$[3,8]$} \\
\hline PEO & 344 & 26.8 & 0.41 & 29.6 & 5.7 & 23.3 & 1.18 & 4.6 & -3.3 & {$[3,6]$} \\
\hline PET & 553 & 55.6 & 3.13 & 3.5 & 42.2 & 117.5 & 3.70 & 2.06 & 5.5 & {$[3,7,9]$} \\
\hline Ny 66 & 553 & 34.2 & 1.78 & 2.0 & 27.1 & 76.8 & 1.98 & 2.05 & 6.2 & {$[3,7]$} \\
\hline PTFE & 605 & 6.9 & 0.745 & 1.1 & - & 37.4 & 0.34 & 2.11 & - & {$[3,5]$} \\
\hline PDMS & 248 & 10.8 & 0.42 & 9.2 & - & 50.1 & 0.23 & 2.8 & - & [4] \\
\hline
\end{tabular}

$\Delta V_{\mathrm{m}}\left(\mathrm{cm}^{3} / \mathrm{g}\right)$ is converted to $\mathrm{J} /\left(\right.$ bar.g) by a relation that $10 \mathrm{~cm}^{3}=1 \mathrm{~J} / \mathrm{bar}$. PE, polyethylene; PP, polypropylene; PB-1, polybutene-1; POM, polyoxymethylcne; PEO, polyethylene oxide; PET, polyethylenetelephtharate; NY66, nylon66; PTFE, polytetrafluoroethylene; PDMS, polydimethyl siloxane.

Table 2

The relation between the discontinuous change at melting point and coefficients in the Landau theory $\Phi=\Phi_{0}+\left\{k_{0}\left(T-T_{0}\right)^{m}\left(P-P_{0}\right)^{n}\right\} \eta^{2}-C \eta^{4}+\left(C^{2} / 4 A^{*}\right) \eta^{6}$

\begin{tabular}{|c|c|c|c|c|c|}
\hline Gap at $T_{\mathrm{m}}$ & $\begin{array}{l}\Delta S_{\mathrm{m}}=S_{\mathrm{m}, 1} \\
-S_{\mathrm{m}, \mathrm{s}}\end{array}$ & $\begin{array}{l}\Delta V_{\mathrm{m}}=V_{\mathrm{m}, 1} \\
-V_{\mathrm{m}, \mathrm{s}}\end{array}$ & $\begin{array}{l}\frac{\Delta C_{P_{\mathrm{n}} \mathrm{n}}}{T_{\mathrm{n} 1}}= \\
\frac{C_{P, \mathrm{~m}, \mathrm{l}}-C_{P_{\mathrm{t}} \mathrm{m}, \mathrm{s}}}{T_{\mathrm{m} 1}}\end{array}$ & $\begin{array}{l}-\Delta\left(\frac{\partial V}{\partial P}\right)_{T, \mathrm{~m}}= \\
-\left(\frac{\partial V}{\partial P}\right)_{T, \mathrm{~m}, 1}+\left(\frac{\partial V}{\partial P}\right)_{T, \mathrm{~m}, \mathrm{~s}}\end{array}$ & $\begin{array}{l}\Delta\left(\frac{\partial V}{\partial T}\right)_{P, \mathrm{~m}}= \\
\left(\frac{\partial V}{\partial T}\right)_{P, \mathrm{~m}, \mathrm{l}}-\left(\frac{\partial V}{\partial T}\right)_{P, \mathrm{~m}, \mathrm{~s}}\end{array}$ \\
\hline Landau theory & $=\frac{2 m \Lambda^{* 2}}{C\left(T_{\mathrm{m}}-T_{0}\right)}$ & $=\frac{2 n A^{* 2}}{C\left(P_{0}-P_{\mathrm{m}}\right)}$ & $=\frac{2 m A^{* 2}(m / 2-1)}{C\left(T_{\mathrm{m}}-T_{0}\right)^{2}}$ & $=\frac{-2 n A^{* 2}(1-n / 2)}{C\left(P_{0}-P_{\mathrm{m}}\right)^{2}}$ & $=\frac{n m A^{* 2}}{C\left(T_{m}-T_{0}\right)\left(P_{0}-P_{m}\right)}$ \\
\hline
\end{tabular}

Table 3

A qualitative relation between the indices $m$ and $n$ in function of $A$ in Eq. (6) and the sign of discontinuous changes at the melting point

\begin{tabular}{lcccc}
\hline & $\Delta S_{\mathrm{m}}$ & $\Delta V_{\mathrm{m}}$ & $\Delta\left(\frac{\partial S}{\partial T}\right)_{P_{\mathrm{m}}}$ & $-\Delta\left(\frac{\partial V}{\partial P}\right)_{T, \mathrm{~m}}$ \\
\hline$a\left(T-T_{0}\right)+b\left(P_{0}-P\right)$ & + & + & - & - \\
$K_{0}\left(T-T_{0}\right)\left(P_{0}-P\right)$ & + & + & - & - \\
$K_{0}\left(T-T_{0}\right)^{2}\left(P_{0}-P\right)^{2}$ & + & + & 0 & + \\
$K_{0}\left(T-T_{0}\right)^{3}\left(P_{0}-P\right)^{3}$ & + & + & + & 0 \\
$K_{0}\left(T-T_{0}\right)^{m}\left(P_{0}-P\right)^{n}, n, m>3$ & + & + & + & + \\
\hline
\end{tabular}

discontinuous change $\Delta Q_{\mathrm{m}}$ are shown in Table 3 where a result calculated for a different type of function of $A$ that $A=a\left(T-T_{0}\right)+b\left(P_{0}-P\right)$ is also shown. The signs of $\Delta(\partial S / \partial T)_{P, \mathrm{~m}},-\Delta(\partial V / \partial P)_{T, \mathrm{~m}}$, and $\Delta(\partial V / \partial T)_{P}, \mathrm{~m}$ predicted by $A=a\left(T-T_{0}\right)+b\left(P_{0}-P\right)$ are all negative as is shown in Table 3, which are inconsistent with the experimental result in Table 1 . On the other hand, in the case of $A=k_{0}\left(T-T_{0}\right)^{m}\left(P_{0}-P\right)^{n}$ the signs of $\Delta(\partial S / \partial T)_{P, \mathrm{~m}}$, and $-\triangle(\partial V / \partial P)_{T, \mathrm{~m}}$ depend on the value of $m$ and $n$ although $\Delta V_{\mathrm{m}}$ and $\triangle(\partial V / \partial T)_{P, \mathrm{~m}}$ are positive for any values of $m$ and $n$. In the case of $m>2$ and $n>2$, the signs of $\Delta(\partial S / \partial T)_{P, m}$ and $-\triangle(\partial V / \partial P)_{T, m}$ are always positive as is shown in Table 3 , while in the case of $m=n=2$ these values are zero and $(\partial S / \partial T)_{P, \mathrm{~m}}$ and $-(\partial V / \partial P)_{T, \mathrm{~m}}$ are continuous at the melting point.

\section{Results}

It is necessary to determine the values of $m$ and $n$ in $A=k_{0}\left(T-T_{0}\right)^{m}\left(P_{0}-P\right)^{n}$ to determine the Gibbs free energy $\Phi$ experimentally. The values of $m$ and $n$ are calculated by relations obtained from by the relations listed in Table 2, which is given by:

$m=\frac{2}{\left[1-\left(\Delta V_{\mathrm{m}} \Delta C_{P, \mathrm{~m}}\right) /\left\{T_{\mathrm{m}} \Delta S_{\mathrm{m}} \Delta(\partial V / \partial T)_{P, \mathrm{~m}}\right\}\right]}$

and

$n=\frac{2}{\left[1-\left(\Delta S_{\mathrm{m}}\left\{-\Delta(\partial V / \partial P)_{T, \mathrm{~m}}\right\} /\left\{\Delta V_{\mathrm{m}} \Delta(\partial V / \partial T)_{P, \mathrm{~m}}\right\}\right)\right]}$

The values of $m$ and $n$ calculated by the experimental data of discontinuous changes in Table 1 are listed in Table 1 where values of $m$ are positive, while values of $n$ are scattered over negative to positive values. An accurate determination of $n$ is quite difficult because the value of $-\Delta(\partial V / \partial P)_{T, \mathrm{~m}}$ in $\mathrm{Eq}$. (11) is very small and order of $10^{-4} \mathrm{~cm}^{3} /(\mathrm{mol}$ bar). Besides, values of $-\Delta(\partial V / \partial P)_{T, m}$ are obtained from the difference at the melting point by an extrapolation of $-(\partial V / \partial P)_{T, m}$ to the melting point from both sides of liquid and crystalline states. That is a main reason why values of $-\Delta(\partial V / \partial P)_{T, m}$ in Table 1 evaluated by different authors are different considerably compared to the other values.

The value of $\Delta(\partial S / \partial T)_{P, \mathrm{~m}}=\Delta C_{P, \mathrm{~m}} / T_{\mathrm{m}}$ is another important quantity which is evaluated from the data of heat capacity 
for crystalline polymer over the temperature range including the melting point [3]. It is found from the experimental data that the derivatives in this work, such as $C_{P, \mathrm{~m}},-(\partial V / \partial P)_{T, \mathrm{~m}}$, and $(\partial V / \partial T)_{P, m}$ for liquid phase are larger than those for solid phase at the melting point, which means that $\Delta Q_{\mathrm{m}}$ is positive. It is interesting to point out that the values of $C$ are positive due to $A^{* 2} / C>0$ as is shown in Table 1 and $A^{*}$ is also positive by relations that $\eta_{\min }^{* 2}=2 A^{*} / C$ and $C>0$.

\section{Discussions}

It is interesting to discuss the discontinuous change of $\gamma_{\mathrm{v}}$ over volumes from crystalline to amorphous state at the melting point from a viewpoint of polymer processing. The thermal pressure coefficient $\gamma_{V}$ has been calculated by a relation that $\gamma_{V}=(\partial V / \partial T)_{P} /\left\{-(\partial V / \partial P)_{T}\right\}$ and is given by:

$\gamma_{\mathrm{v}, \mathrm{s}}=\gamma_{\mathrm{v}, \mathrm{m}, 1}-\frac{\left.\left(n A^{* 2} / C\right)\left(1-\Delta V / \Delta V_{\mathrm{m}}\right)\left\{m(3 Z-2)\left(P_{0}-P\right)+2\left(T_{\mathrm{m}}-T_{0}\right) \gamma_{\mathrm{v}, \mathrm{m}, 1} Z+n(2-3 Z) / 2\right\}\right\}}{\left(T_{\mathrm{m}}-T_{0}\right)\left\{Z\left(P_{0}-P_{\mathrm{m}}\right)^{2}\left\{-(\partial V / \partial P)_{T, \mathrm{~m}, 1}\right\}+2\left(n A^{* 2} / C\right)\left(1-\Delta V / \Delta V_{\mathrm{m}}\right)\{Z+n(2-3 Z) / 2\}\right\}}$

The behavior of $\gamma_{\mathrm{V}}$ at the melting transition is estimated through a variable expressed by $\Delta \gamma_{v}=\gamma_{v, s}-\gamma_{v, m}$. The discontinuous change of $\gamma_{v}$ at the melting point $\Delta \gamma_{v}$ is calculated by $\Delta \gamma_{\mathrm{V}}=\gamma_{\mathrm{v}, \mathrm{m}, 1}-\gamma_{\mathrm{v}, \mathrm{m}, \mathrm{s}}-\left(\gamma_{\mathrm{v}, \mathrm{m}, 1}-\gamma_{\mathrm{v}, \mathrm{s}}\right)$, where $\gamma_{\mathrm{v}, \mathrm{m}, \mathrm{l}}-\gamma_{\mathrm{v}, \mathrm{m}, \mathrm{s}}=\Delta \gamma_{\mathrm{v}, \mathrm{m}}$ is obtained from Eq. (A.15) and $\left(\gamma_{\mathrm{v}, \mathrm{m}, 1}-\gamma_{\mathrm{v}, \mathrm{s}}\right)$ is obtained from Eq. (12).

It is important to examine the original function of $\Phi$ at the melting point in Eq. (1), which is rewritten as

$\Phi=\Phi_{0}+\left(a_{0} C\right)^{1 / 2} \eta^{2}-C \eta^{4}+\left\{\frac{C\left(C / a_{0}\right)^{1 / 2}}{4}\right\} \eta^{6}$

In deriving Eq. (13) following relations that $E=C^{2} / 4 A^{*}$ and $A^{* 2} / C=a_{0}$ are used. A relation $\left(A^{* 2} / C\right)=\Delta S_{\mathrm{m}} \Delta V_{\mathrm{m}} /\left\{4 \Delta(\partial V / \partial T)_{P, \mathrm{~m}}\right\}$ is used to evaluate the $\left(A^{* 2} / C\right)$ in Table 1. The typical function of $\Phi$ for PDMS with respect to $C$ and $\eta$ at $T=T_{\mathrm{m}}$ is shown in Fig. 1 where two minimum points at $\eta=0$ and $\eta=\eta_{\min }^{*}$ are indicated where $\Phi_{0}(\eta=0)$ is equal to $\Phi\left(\eta_{\min }^{*}\right)=\Phi_{0}(\eta=0)$.
The other values in the Landau theory such as $\left(T_{\mathrm{m}}-T_{0}\right)$ and $\left(P_{0}-P_{\mathrm{m}}\right)$ are evaluated from the experimental data of ratios of discontinuous gaps in Table 1 and relations in Table 4, where $\Delta X$ for the line corresponds to $\Delta S_{\mathrm{m}}$, $\Delta V_{\mathrm{m}}, \Delta(\partial S / \partial T)_{P, \mathrm{~m}}$ and $-\Delta(\partial V / \partial P)_{T, \mathrm{~m}}$ while $\Delta Y$ for the row also corresponds to $\Delta S_{\mathrm{m}}, \Delta V_{\mathrm{m}}, \Delta(\partial S / \partial T)_{P, \mathrm{~m}}$ and $-\Delta(\partial V / \partial P)_{T, \mathrm{~m}}$. The values listed in Table 4 give the ratios of the discontinuous change $\Delta X / \Delta Y$. The value of $\left(T_{\mathrm{m}}-T_{0}\right)$ is calculated by $(m / 2-1) \Delta S_{\mathrm{m}} / \Delta(\partial S / \partial T)_{P}, \mathrm{~m}$ using the relation that $\Delta S_{\mathrm{m}} / \Delta(\partial S / \partial T)_{P, \mathrm{~m}}=\left(T_{\mathrm{m}}-T_{0}\right) /(m / 2-1)$ in Table 4. In the case of PDMS with $T_{\mathrm{m}}=248 \mathrm{~K}$, it is obtained that $\left(T_{\mathrm{m}}-T_{0}\right)=116 \mathrm{~K}$ and $T_{0}=132 \mathrm{~K}$. It is obvious that the experimental values, such as $\Delta V_{\mathrm{m}}$ and $\Delta S_{\mathrm{m}}$ can reproduce or predict by using equations in Table 2 and values of $m, n$, $\left(A^{* 2} / C\right)$ in Table 1 because the experimental values of $\Delta V_{\mathrm{m}}$, $\Delta S_{\mathrm{m}}$ and others are used to evaluate $m, n,\left(A^{* 2} / C\right)$ and other values in the $\Phi$ function.
It is interesting to point out that the Landau theory of phase transition [2] predicts a metastable region in the melting transition. The metastable state appears over a temperature between $T_{2}$ and $T_{0}$ where $T_{2}$ is a temperature at which the minimum value of order parameter $\eta_{\min }^{2}$ is equal to the maximum value of $\eta_{\max }^{2}$ and therefore a relation $C^{2}=3 A E$ is obtained. The equation for $T_{2}$ at $P=P_{\mathrm{m}}$ is given using relations that $E=C^{2} / 4 A^{*}$ and $C^{2}=3 A E$ by:

$T_{2}=T_{0}+\left(T_{\mathrm{m}}-T_{0}\right)\left(\frac{4}{3}\right)^{1 / m}$

The solid phase is metastable or less stable than the liquid state over the temperature range $T_{\mathrm{m}}<T \leqq T_{2}$, while the liquid state is metastable or less stable than the solid state over $T_{0} \leqq T<T_{\mathrm{m}}$. The solid and liquid phases are equally stable at $T_{\mathrm{m}}$ where $\Phi_{0}(\eta=0)=\Phi\left(\eta_{\min }^{*}\right)$ and a relation between the

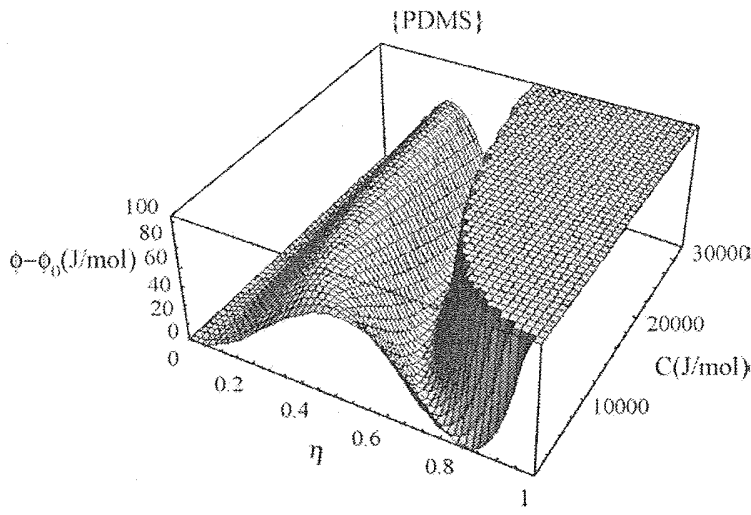

Fig. 1. Typical $\left(\Phi-\Phi_{0}\right)-\eta-C$ function at the melting transition calculated by Eq. (13) with $\mathrm{a}_{0}=230 \mathrm{~J} / \mathrm{mol}$ for poly (dimethyl siloxane) (PDMS). 
Table 4

The ratios of the discontinuous change $\Delta X / \Delta Y$ is expressed by the coefficients in the Landau theory where $\Delta X$ is the value for the line, while $\Delta Y$ is that for the row

\begin{tabular}{|c|c|c|c|c|}
\hline \multirow[t]{2}{*}{$\Delta Y$} & \multicolumn{4}{|l|}{$\Delta X$} \\
\hline & $\Delta S_{\mathrm{m}}$ & $\Delta V_{\mathrm{m}}$ & $\Delta\left(\frac{\partial S}{\partial T}\right)_{P, \mathrm{~m}}$ & $-\Delta\left(\frac{\partial V}{\partial P}\right)_{T, \mathrm{~m}}$ \\
\hline$\Delta S_{\mathrm{m}}$ & 1 & & & \\
\hline$\Delta V_{\mathrm{m}}$ & $\frac{m\left(P_{0}-P_{\mathrm{m}}\right)}{n\left(T_{\mathrm{m}}-T_{0}\right)}$ & 1 & & \\
\hline$\Delta\left(\frac{\partial S}{\partial T}\right)_{P, \mathrm{~m}}$ & $\frac{T_{\mathrm{m}}-T_{0}}{m / 2-1}$ & $\frac{n\left(T_{m}-T_{0}\right)^{2}}{m(m / 2-1)\left(P_{0}-P_{m}\right)}$ & 1 & \\
\hline$-\Delta\left(\frac{\partial V}{\partial P}\right)_{T, \mathrm{~m}}$ & $\frac{-m\left(P_{0}-P_{\mathrm{m}}\right)^{2}}{n(1-n / 2)\left(T_{\mathrm{m}}-T_{0}\right)}$ & $\frac{-\left(P_{0}-P_{\mathrm{m}}\right)}{1-n / 2}$ & $\frac{m(m / 2-1)\left(P_{0}-P_{\mathrm{m}}\right)^{2}}{-n(1-n / 2)\left(T_{\mathrm{m}}-T_{0}\right)^{2}}$ & 1 \\
\hline$\Delta\left(\frac{\partial V}{\partial T}\right)_{P, \mathrm{~m}}$ & $\frac{2\left(P_{0}-P_{\mathrm{m}}\right)}{n}$ & $\frac{2\left(T_{\mathrm{m}}-T_{0}\right)}{m}$ & $\frac{2(m / 2-1)\left(P_{0}-P_{\mathrm{m}}\right)}{n\left(T_{\mathrm{m} 1}-T_{0}\right)}$ & $\frac{-2(1-n / 2)\left(T_{\mathrm{m}}-T_{0}\right)}{m\left(P_{0}-P_{\mathrm{m}}\right)}$ \\
\hline
\end{tabular}

For example $\Delta X / \Delta Y=\Delta S_{\mathrm{m}} / \Delta V_{\mathrm{m}}=m\left(P_{0}-P_{\mathrm{m}}\right) / n\left(T_{\mathrm{m}}-T_{0}\right)$.

temperature and order parameter $\eta_{\min }$ is given using Eq. (5) by:

$$
T=T_{0}+\left(T_{\mathrm{m}}-T_{0}\right)\left[\left(\frac{C}{A^{*}}\right) \eta_{\min }^{2}\left\{2-\left(\frac{3 C}{4 A^{*}}\right) \eta_{\min }^{2}\right\}\right]^{1 / m}
$$

where a power law between $\left(T-T_{0}\right)$ and $\eta_{\min }^{2 / m}$ is suggested.

According to the crystalline-amorphous composite model [12] the discontinuous change $\Delta Q_{\mathrm{m}}$ at the melting transition for the completely crystallized polymer with the degree of crystallinity $x_{\mathrm{c}}=1.0$ is related to that in a semi-crystalline polymer with value of $x_{\mathrm{c}}$ by:

$\Delta Q_{\mathrm{m}}\left(x_{\mathrm{c}}\right)=x_{\mathrm{c}} \Delta Q_{\mathrm{m}}, x_{\mathrm{c}}=1.0$

The ratio of two $\Delta Q_{\mathrm{m}}$ such as $\Delta Q_{1, \mathrm{~m}}\left(x_{\mathrm{c}}\right) / \Delta Q_{2, \mathrm{~m}}\left(x_{\mathrm{c}}\right)$ for a semi-crystalline polymer with $x_{\mathrm{c}}$ is equal to the ratio of $\Delta Q_{1}, \mathrm{~m}\left(x_{\mathrm{c}}=1\right) / \Delta Q_{2, \mathrm{~m}}\left(x_{\mathrm{c}}=1\right)$ for completely crystalline one by the Eq. (16). Therefore, the ratio is independent on $x_{\mathrm{c}}$ and is a quantity reflecting the intrinsic properties of the crystalline polymer if the model is correct. There are many ratios of discontinuous changes at the melting point, if the values $\Delta C_{P, \mathrm{~m}} / T_{\mathrm{m}}, \triangle(\partial V / \partial T)_{p}, \mathrm{~m}$ and $-\triangle(\partial V / \partial P)_{T, \mathrm{~m}}$ are taken into account in addition to $\Delta V_{\mathrm{m}}$ and $\Delta S_{\mathrm{m}}$. The ratio $\Delta V_{\mathrm{m}} / \Delta S_{\mathrm{m}}\left(=\mathrm{d} T_{\mathrm{m}} / \mathrm{d} P\right)$ is one of the typical examples. The relations between these ratios and the coefficients in the Landau theory summarized in Table 4 show that this ratio does not depend on $C$ and $A^{*}$, while the $\triangle Q_{\mathrm{m}}$ depends on $C$ and $A^{*}$ as is shown in Table 2. It is noteworthy that a relation $\Delta V_{\mathrm{m}}=\left\{n A^{*} /\left(P_{0}-P_{\mathrm{m}}\right)\right\} \eta_{\min }^{* 2}$ derived from Eqs. (A.6) and (A.10) suggests that all of discontinuous change at $T_{\mathrm{m}}$ in Table 2 is proportional to $A^{*} \eta_{\text {min }}^{* 2}$.

\section{Conclusions}

In this work, the order parameter $\eta$ is introduced to take into account a change of degree of order of polymer during the melting transition based on the Landau theory which can predict the melting transition or the first order phase transition successfully. We have determined the function of $\eta$ with respect to volume of solid $V_{\mathrm{S}}$ based on the Landau theory. A general criterion for the signs of discontinuous changes such as $\Delta \gamma_{\mathrm{v}, \mathrm{m}}, \Delta(\partial V / \partial T)_{P, \mathrm{~m}},-\Delta(\partial V / \partial P)_{T, \mathrm{~m}}$ has been discussed in the framework of the Landau theory where $\Delta Q_{\mathrm{m}}$ is defined by $\Delta Q_{\mathrm{m}}=Q_{\mathrm{m}, 1}-Q_{\mathrm{m}, \mathrm{s}}$. The parameters in the Gibbs free energy function $\Phi$ in the Landau theory have been evaluated numerically and a typical function of $\Phi$ for a polymer has been demonstrated as a function of $\eta$ and $C$ at the melting transition. It is also interesting to point out that the results obtained in this work are applicable to the melting phenomena for simple solids with low molecular weight.

\section{Appendix A. Derivation of Eq. (3)}

A definition of $\Phi$ is given by:

$\Phi=\Phi_{0}+A \eta^{2}-C \eta^{4}+E \eta^{6}$

and the minimum condition of $\Phi$ is given by:

$A-2 C \eta^{2}+3 E \eta^{4}=0$

The larger value of $\eta$ in Eq. (2) gives the minimum of $\Phi$, while the smaller value of $\eta$ gives the maximum of $\Phi$ and are expressed by:

$\eta_{\min }^{2}=\frac{C+\left(C^{2}-3 A E\right)^{1 / 2}}{3 E}$

and

$\eta_{\max }^{2}=\frac{C-\left(C^{2}-3 A E\right)^{1 / 2}}{3 E}$ 
By substitution of Eq. (A.1) into Eq. (1) the minimum function of $\Phi$ is obtained:

$$
\begin{aligned}
\Phi_{\min }= & \Phi_{0}+\frac{\left\{C+\left(C^{2}-3 A E\right)^{1 / 2}\right\}\left\{2 A / 3-2 C^{2} / 9 E\right\}}{3 E} \\
& +\frac{A C}{9 E}
\end{aligned}
$$

The conditions at the melting point are given by $\Phi=\Phi_{0}$ and the minimum condition of $\Phi$ in Eq. (2). The former condition is given by:

$A^{*} \eta_{\min }^{* 2}-C \eta_{\min }^{* 4}+E \eta_{\min }^{* 6}=0$

and the latter one is given by:

$A^{*}-2 C \eta_{\min }^{* 2}+3 E \eta_{\min }^{* 4}=0$

It is obtained from Eqs. (A.4) and (A.5) that:

$\eta_{\min }^{* 2}=\frac{2 A^{*}}{C}$

and

$E=\frac{C^{2}}{4 A^{*}}$

We assumed in this work that $E$ is constant and given by Eq. (A.7). By substituting $E=C^{2} / 4 A^{*}$ into Eq. (A.3), a following equation is obtained:

$$
\begin{aligned}
\Phi_{\min }=\Phi_{0} & +\left(\frac{4 A^{* 2}}{3 C}\right)\left[\left(\frac{A}{A^{*}}\right)-\left(\frac{8}{9}\right)\right. \\
& \left.+\left(4-\frac{3 A}{A^{*}}\right)^{1 / 2}\left(\frac{A}{3 A^{*}}-\frac{4}{9}\right)\right]
\end{aligned}
$$

By introducing a parameter $Z=\left(4-3 A / A^{*}\right)^{1 / 2}$ the $\mathrm{Eq}$. (A.8) is given by:

$\Phi_{\min }=\Phi_{0}+\left(\frac{4 A^{* 2}}{27 C}\right)\left[4-3 Z^{2}-Z^{3}\right]$
Appendix $B$. Derivation of $\Delta(\partial V / \partial T)_{P},-\Delta(\partial V / \partial P)_{T}$, and $\Delta(\partial P / \partial T)_{V}$ at melting point

The volume of solid $V_{s}$ calculated from $\left(\partial \Phi_{\min } / \partial P\right)_{T}$ is given by:

$V_{\mathrm{s}}=V_{\mathrm{m}, 1}-\frac{\left(2 A^{* 2} n / 9 C\right)(2+Z)\left(4-Z^{2}\right)}{P_{0}-P_{\mathrm{m}}}$

and $\Delta V_{\mathrm{m}}=V_{\mathrm{m}, \mathrm{l}}-V_{\mathrm{m}, \mathrm{s}}$ is given by:

$\Delta V_{\mathrm{m}}=\frac{2 A^{* 2} n / C}{P_{0}-P_{\mathrm{m}}}$

The relation for $\Delta V=V_{\mathrm{s}}-V_{\mathrm{m}, \mathrm{s}}$ at $P=P_{\mathrm{m}}$ is expressed using Eqs. (A.9) and (A.10) by:

$\Delta V_{\mathrm{m}}-\Delta V=\frac{\Delta V_{\mathrm{m}}(2+Z)\left(4-Z^{2}\right)}{9}$

There are three solutions of $Z$ for a $\Delta V$. A positive value of $Z$ is obtained for $\Delta V>\Delta V_{\mathrm{m}} / 9$ and two positive $Z$ are obtained for $0 \leq \Delta V \leq \Delta V_{\mathrm{m}} / 9$, while $Z=0$ and $\left(5^{1 / 2}-1\right)$ for $\Delta V=\Delta V_{\mathrm{m}} / 9$. The other value of $Z$ is negative or complex number.

The thermal expansion coefficient $\alpha$ and the compressibility $\beta$ are calculated from Eq. (A.9) and are given by:

$$
\begin{aligned}
\left(\frac{\partial V}{\partial T}\right)_{P, \mathrm{~s}}= & \left(\frac{\partial V}{\partial T}\right)_{P, \mathrm{~m}, 1} \\
& -\frac{\left(n m A^{* 2} / C\right)\left(1-\Delta V / \Delta V_{\mathrm{m}}\right)\{(3 Z-2) / Z\}}{\left(P_{0}-P\right)\left(T-T_{0}\right)}
\end{aligned}
$$

and

$$
\begin{aligned}
& -\left(\frac{\partial V}{\partial P}\right)_{T, \mathrm{~s}}=-\left(\frac{\partial V}{\partial P}\right)_{T, \mathrm{~m}, \mathrm{I}} \\
& +\frac{\left(2 n A^{* 2} / C\right)\left(1-\Delta V / \Delta V_{\mathrm{m}}\right)\{1+n(2-3 Z) / 2 Z\}}{\left(P_{0}-P\right)^{2}}
\end{aligned}
$$

The thermal pressure coefficient $\gamma_{V}$ is given using $\gamma_{V}=(\partial V / \partial T)_{P} /-(\partial V / \partial P)_{T}$ by:

$$
\gamma_{\mathrm{v}, \mathrm{s}}=\gamma_{\mathrm{v}, \mathrm{m}, 1}-\frac{\left(n A^{* 2} / C\right)\left(1-\Delta V / \Delta V_{m}\right)\left\{m(3 Z-2)\left(P_{0}-P\right)+2\left(T-T_{0}\right) \gamma_{\mathrm{v}, \mathrm{m}, 1}\{Z+n(2-3 Z) / 2\}\right\}}{\left(T-T_{0}\right)\left\{Z\left(P_{0}-P\right)^{2}\left\{-(\partial V / \partial P)_{T, \mathrm{~m}, 1}\right\}+2\left(n A^{* 2} / C\right)\left(1-\Delta V / \Delta V_{\mathrm{m}}\right)\{Z+n(2-3 Z) / 2\}\right\}}
$$

The $\gamma_{\mathrm{v}, \mathrm{m}, \mathrm{s}}$ is obtained by using Eq. (14) with $\Delta V=0$. At the melting point $T=T_{\mathrm{m}}$ and $P=P_{\mathrm{m}}$ where $\Delta V=\Delta V_{\mathrm{m}}$ and $Z=1, \Delta \gamma_{v, m}$ is given by:

$$
\Delta \gamma_{\mathrm{v}, \mathrm{m}}=\gamma_{\mathrm{v}, \mathrm{m}, 1}-\gamma_{\mathrm{v}, \mathrm{m}, \mathrm{s}}=\frac{\left(n A^{* 2} / C\right)\left\{m\left(P_{0}-P_{\mathrm{m}}\right)+2\left(T_{\mathrm{m}}-T_{0}\right) \gamma_{\mathrm{v}, \mathrm{m}, 1}(1-n / 2)\right\}}{\left(T_{\mathrm{m}}-T_{0}\right)\left\{\left(P_{0}-P_{\mathrm{m}}\right)^{2}\left\{-\left(\partial V_{1} / \partial P\right)_{T}\right\}+2\left(n A^{* 2} / C\right)(1-n / 2)\right\}}
$$




\section{Appendix $\mathrm{C}$. Derivation of discontinuous change of heat capacity $\triangle C_{P}, \mathrm{~m}$ at melting point}

The entropy for solid state $S_{\mathrm{s}}$ is given using $\Phi_{\min }$ in $\mathrm{Eq}$. (3) and $\left(\partial \Phi_{\min } / \partial T\right)=-S$ by:

$S_{\mathrm{s}}=S_{\mathrm{m}, 1}-\frac{\left(2 m A^{* 2} / C\right)\left(1-\Delta V / \Delta V_{\mathrm{m}}\right)}{T_{\mathrm{m}}-T_{0}}$

where $S_{\mathrm{m}, 1}$ is $S$ for liquid phase at the melting point. The $S_{\mathrm{m}, \mathrm{s}}$ for solid phase at $\left(T_{\mathrm{m}}, P_{\mathrm{m}}\right.$ is give by Eq. (A.16) with $\Delta V=0$ or $V_{\mathrm{s}}=V_{\mathrm{m}, \mathrm{s}}$ which is given:

$S_{\mathrm{m}, \mathrm{s}}=S_{\mathrm{m}, 1}-\frac{2 m A^{* 2} / C}{T_{\mathrm{m}}-T_{0}}$

The entropy change $\Delta S_{\mathrm{m}}$ at the melting point is given by:

$\Delta S_{\mathrm{m}}=\frac{2 m A^{* 2} / C}{T_{\mathrm{m}}-T_{0}}$

while the $\triangle(\partial S / \partial T)_{P, \mathrm{~m}}\left(=\left(C_{P, \mathrm{~m}, \mathrm{1}}-C_{P, \mathrm{~m}, \mathrm{~s}}\right) / T_{\mathrm{m}}\right)$ is given by:

$$
\begin{aligned}
& \frac{C_{P, \mathrm{~m}, 1}-C_{P, \mathrm{~s}}}{T_{\mathrm{m}}} \\
& =\frac{\left(2 A^{* 2} m / C\right)\left(1-\Delta V / \Delta V_{\mathrm{m}}\right)(3 m / 2-1-m / Z)}{\left(T-T_{0}\right)^{2}}
\end{aligned}
$$

The discontinuous change of $C_{P}, \mathrm{~m}$ at the melting point $\triangle C_{P, \mathrm{~m}}$ is given by:

$\frac{\Delta C_{P, \mathrm{~m}}}{T_{\mathrm{m}}}=\frac{C_{P, \mathrm{~m}, 1}-C_{P, \mathrm{~m}, \mathrm{~s}}}{T_{\mathrm{m}}}=\frac{\left(2 A^{* 2} m / C\right)(m / 2-1)}{\left(T_{\mathrm{m}}-T_{0}\right)^{2}}$

where $C_{P}$ is the heat capacity at constant pressure.

\section{Appendix D. Calculation for a case of}

$A=a\left(T-T_{0}\right)+b\left(P_{0}-P\right)$

A simple function for $A$ is given by:

$A=a\left(T-T_{0}\right)+b\left(P_{0}-P\right)$

The $A^{*}$ at the melting point is given by:

$A^{*}=a\left(T_{\mathrm{m}}-T_{0}\right)+b\left(P_{0}-P_{\mathrm{m}}\right)$

where coefficient of $a$ and $b$ are constant, while $T_{0}$ and $P_{0}$ are temperature and pressure at $A=0$, respectively. The function of $\Phi_{\min }$ is given by:

$\Phi_{\min }=\Phi_{0}+\left(\frac{4 A^{* 2}}{27 C}\right)\left(4-3 Z^{2}-Z^{3}\right)$
The volume $V_{\mathrm{s}}$ in ordered state is given from Eq. (A.23) by:

$V_{\mathrm{s}}=V_{\mathrm{m}, 1}-\left(\frac{2 A^{*} b}{3 C}\right)(2+Z)$

where $(\partial Z / \partial P)_{T}=3 b /\left(2 Z A^{*}\right)$ is used. The volume change at the melting $\Delta V_{\mathrm{m}}$ is given using $Z=1$ or $A=A^{*}$ by:

$\Delta V_{\mathrm{m}}=V_{\mathrm{m}, 1}-V_{\mathrm{m}, \mathrm{s}}=\left(\frac{2 A^{*} b}{C}\right)$

The entropy at ordered state is given by:

$S_{\mathrm{s}}=S_{\mathrm{m}, 1}-\left(\frac{2 A^{*} a}{3 C}\right)(2+Z)$

where $(\partial Z / \partial T) p=-3 a /\left(2 Z A^{*}\right)$ is used. The entropy change $\Delta S_{\mathrm{m}}$ at melting is given by:

$\Delta S_{\mathrm{m}}=S_{\mathrm{m}, 1}-S_{\mathrm{m}, \mathrm{s}}=\left(\frac{2 A^{*} a}{C}\right)$

The $(\partial S / \partial T)_{P, \mathrm{~s}}$ at the ordered state is given by:

$\left(\frac{\partial S}{\partial T}\right)_{P, \mathrm{~s}}=\left(\frac{\partial S}{\partial T}\right)_{P, \mathrm{~m}, 1}+\frac{a^{2}}{C Z}$

and the change of $(\partial S / \partial T)_{P}$ at the transition point is given by:

$\left(\frac{\partial S}{\partial T}\right)_{P, \mathrm{~m}, \mathrm{~s}}=\left(\frac{\partial S}{\partial T}\right)_{P, \mathrm{~m}, 1}+\frac{a^{2}}{C}$

and is rewritten as:

$\left(\frac{\partial S}{\partial T}\right)_{P, \mathrm{~m}, \mathrm{l}}-\left(\frac{\partial S}{\partial T}\right)_{P, \mathrm{~m}, \mathrm{~s}}=\frac{\Delta C_{P, \mathrm{~m}}}{T_{\mathrm{m}}}=\frac{-a^{2}}{C}$

The similar calculation for $\Delta(\partial V / \partial T)_{P, \mathrm{~m}}$, and $-\Delta(\partial V / \partial P)_{T, \mathrm{~m}}$ has been done by using Eq. (A.24).

\section{References}

[1] L.D. Landau, E.M. Lifshitz, Statistical Physics, Pergamon Press, Oxford, 1958.

[2] J.-C. Toledano, P. Toledano, The Landau theory of Phase Transitions, World Scientific Publishing, 1987

[3] J. Brandrup, E.H. Immergut, Polymer Handbook, Wiley, New York, 1989.

[4] F.E. Karasz, P.R. Couchmann, D. Klempner, Macromolecules 10 (1977) 88-89.

[5] P. Zoller, J. Appl. Polym. Sci. 22 (1978) 633-641.

[6] W. Li, M. Radosz, Macromolecules 26 (1993) 1417-1423.

[7] J. He, P. Zoller, J. Polym. Sci., Part B: Polym. Phys. 32 (1994) 1049-1067.

[8] H.W. Starkweather Jr., G.A. Jones, J. Polym. Sci., Part B: Polym. Phys. 26 (1988) 257-266.

[9] P. Zoller, J. Macromol. Sci.: Phys. B 18 (3) (1980) 555 568.

[10] P. Zoller, J. Appl. Polym. Sci. 23 (1979) 1057-1061.

[11] O. Olabisi, R. Simha, Macromolecules 8 (1975) 206210.

[12] S. Matsuoka, J. Polym. Sci. 57 (1962) 569-588. 